\title{
Nominata de Pareceristas
}

\author{
Alexandre Nogueira Martins \\ Aline Passuelo De Oliveira \\ Angelica Carvalho \\ Bianca Suzy Dos Reis Dos Santos \\ Bruno Cardoso \\ Bruno Domingues \\ Carlos Henrique Moraes Dos Santos \\ Caroline Pacheco \\ Claudia Santos \\ Daniel Brochado Pires \\ Edson Mendes Nunes Júnior \\ Érika Guimarães Ferreira \\ Eumar Andre Kohler \\ Fabio Augusto Souza \\ Fabricio Teló \\ Felipe Serafim Vieira \\ Fernando Diehl \\ Gabriel Carneiro \\ Ísis De Jesus Garcia \\ Juliana Inez Luiz De Souza \\ Kamille Mattar \\ Lenin Bicudo Bárbara \\ Leonardo Esteves \\ Liciane Barbosa De Mello \\ Luanna Fernanda Da Cruz Bach \\ Lucas Carvalho \\ Lucas Maciel \\ Luciano Dutra De Oliveira \\ Lígia Wilhelms Eras \\ Marcela Guedes \\ Marcio Bernardes De Carvalho \\ Marco Rossi \\ Marcos Meinerz \\ Maria Rita Mattar \\ Mauricio Priess Da Costa \\ Ramiro Garcia \\ Rodrigo Cristiano Diehl \\ Sandra Maria Mattar Diaz \\ Tânia Bittencourt Bloomfield \\ Urbano Lemos Júnior \\ Valeska Berman Machado \\ Vitor Hugo Da Silva Adami \\ Wallace Cabral Ribeiro
}

